\title{
Psicofármacos e VIH
}

\section{Psychotropic Drugs and HIV}

Ana-Lúcia Moreira ${ }^{*, * *}{ }^{-}$, Melinda Carmen Godinho Pereira ${ }^{* *}$, Diogo Telles-Correia ${ }^{*, * *, * * * *}$

\section{RESUMO:}

Introdução: A infecção VIH/SIDA está muitas vezes associada a perturbações psiquiátricas tais como psicose, depressão e ansiedade. Comorbilidades psiquiátricas podem interferir na adesão à terapêutica anti-retrovírica, pelo que o seu diagnóstico e o tratamento oportuno são essenciais. No entanto, a administração de um psicofármaco concomitante à terapêutica HAART pode resultar em interacções medicamentosas.

Objectivos: Esta revisão pretende analisar os vários psicofármacos que podem ser usados nestes doentes, bem como as interacções e reacções adversas que podem surgir.

Métodos: Foi efectuada uma pesquisa na literatura anglo-saxónica, de 1993 até 2011, pela MEDLINE, utilizando como palavras-chave: HIV, AIDS, psychosis, depression, anxiety, secondary mania, antidepressive agents, antipsychotics, benzodiazepines, HAART.

Resultados: Foram localizados 100 artigos, dos quais 66 foram incluídos e 34 excluídos. Os artigos que não apresentassem dados específicos sobre o uso de psicofármacos nos doentes com VIH foram excluídos.

Discussão: Podem ocorrer interações farmacológicas por ocupação das mesmas vias de metabolização dos medicamentos. São necessários estudos adicionais com indicações para uma boa prática clínica. São ainda de considerar as intervenções psicoterapêuticas.

Conclusão: A escolha da intervenção terapêutica, nomeadamente quando se consideram psicofármacos, com o menor número de interacções e efeitos adversos é crucial para alcançar 0 sucesso terapêutico no tratamento dos doentes com VIH.

Palavras-Chave: Psicofármacos; VIH; Anti-retrovirais; Interações Medicamentosas.

\section{ABSTRACT:}

Background: HIV/AIDS infection is frequently associated with psychiatric disorders like psychosis, depression and anxiety. Psychiatric comorbidities may interfere with adherence to antiretroviral treatment. Therefore, diagnosis and treatment of these conditions are essential. However, the administration of a psychotropic drug to HAART therapy can result in drug interactions.

Objectives: This review aims to analyze the various psychotropic drugs that can be used in these patients, as well as the interactions and adverse reactions that may occur.

\footnotetext{
*Centro Hospitalar Lisboa Norte, EPE; **Faculty of Medicine of the University of Lisbon; ***:Psychiatric approach to Liver Transplanted Patients' Unit of Curry Cabral Hospital's Liver Transplantation Center, Lisbon, Portugal. \ almoreira@campus.ul.pt

Recebido/Received: 06/06/2013 - Aceite / Accepted: 09/12/2013
}

Revista do Serviço de Psiquiatria do Hospital Prof. Doutor Fernando Fonseca, EPE 
Methods: A MEDLINE search on anglo-saxonic literature was conducted, from 1993 until 2011, using the key-words: HIV, AIDS, psychosis, depression, anxiety, secondary mania, antidepressive agents, antipsychotics, benzodiazepines, HAART.

Results: We found 100 articles, of which 66 were included and 34 excluded. The articles that showed no specific data on the use of psychotropic drugs in HIV patients were excluded.

Discussion: Pharmachologic interactions may occur by occupation of the same metabolic pathways. Further research is needed with indications for best practices. Psychotherapeutic interventions should be considered.

Conclusion: The choice of the therapeutic intervention, namely when considering psychotropic drugs with the lowest number of interactions and adverse effects is crucial in order to achieve therapeutic success in the treatment of HIV infected patients.

Key-Words: Psychotropic Drugs; HIV; Anti-HIV Agents; Drug Interactions.

\section{INTRODUÇÃO}

0 VIH (vírus da imunodeficiência humana) é 0 agente infeccioso causador de SIDA (Síndrome da Imunodeficiência Adquirida) ${ }^{1}$. 0 VIH apresenta uma especificidade de infecção para células humanas ${ }^{2,3}$. As glicoproteínas gp120 e gp41, situadas na superfície viral, são as estruturas que vão ligar 0 VIH às células que tenham receptores compatíveis, nomeadamente os linfócitos CD44. Há, no entanto, outras células que também possuem receptores $\mathrm{CD} 4$ e que são infectadas: os macrófagos, monócitos e células da glia, daí resultando a disseminação do vírus para outros órgãos e, em especial, para 0 Sistema Nervoso Central (SNC) ${ }^{3}$.

A Organização Mundial de Saúde (OMS) estima que existiam cerca de 34 milhões de pessoas no mundo infectadas com VIH em $2011^{5}$. Os perfis de transmissão variam de região para região, consoante factores biológicos e socioculturais ${ }^{6}$. 0 tratamento actual da infecção VIH fundamenta-se no uso de fármacos anti-retrovirais específicos e antibióticos, utilizados para 0 controlo de infecções oportunistas? ${ }^{7}$. Existem actualmente quatro grupos de anti-retrovirais: inibidores da protease (IP's), análogos não nucleósidos inibidores da transcriptase reversa (NNRTI's), análogos nucleósidos inibidores da transcriptase reversa (NRTI's) e inibidores da fusã $0^{8}$. A terapêutica anti-retrovírica é baseada na combinação destes, que internacionalmente ficou conhecida como bighly active antiretroviral therapy (HAART) ${ }^{9} .0$ impacto benéfico da terapêutica anti-retrovírica, evidenciou-se pelo decréscimo acentuado de mortes por SIDA registado $0^{10}$.

No decurso da infecção VIH, o aparecimento de comorbilidade psiquiátrica é bastante comum, oscilando entre 30 a $60 \%{ }^{11}$. Por um lado, pode haver história psiquiátrica prévia: antecedentes de ansiedade, depressão ou abuso de substâncias, que no momento da infecção poderão ressurgir ${ }^{12}$. Por outro lado, a própria acção do VIH no cérebro também é responsável pelo aparecimento de perturbações psiquiátricas, tanto por acção directa do vírus sob o SNC como por efeito adverso da terapêutica anti-retrovírica ${ }^{13}$. Estas podem influenciar a progressão da doença para 0 estadio de $\operatorname{SIDA}^{14}$. 
Após identificada uma comorbilidade psiquiátrica, será com frequência necessário administrar um psicofármaco em conjunto com o esquema terapêutico HAART ${ }^{15}$. Surge então o problema das interacções medicamentosas pois determinados anti-retrovirais são metabolizados pelos mesmos conjuntos de enzimas que participam na hidroxilação, desmetilação ou glucuronidação de psicofármacos, sobretudo antipsicóticos e antidepressivos ${ }^{16,17}$. Além disso, os doentes com VIH têm uma sensibilidade aumentada aos efeitos adversos dos psicofármacos ${ }^{15}$.

Esta revisão tem como objectivo abordar os vários psicofármacos disponíveis para 0 tratamento das perturbações psiquiátricas em doentes com VIH, dando especial ênfase às possíveis interacções entre psicofármacos e anti-retrovirais.

\section{MÉTODOS}

Foi efectuada uma pesquisa na literatura anglo-saxónica, de 1993 até 2011, pela MEDLINE, utilizando como palavras-chave: HIV, AIDS, psychosis, depression, anxiety, secondary mania, antidepressive agents, antipsychotics, benzodiazepines, HAART.

\section{RESULTADOS}

Foram localizados 100 artigos, incluindo revisões, estudos transversais e estudos longitudinais, dos quais 66 foram incluídos e 34 excluídos. Foram incluídas revisões sistemáticas e artigos originais com controlo e randomizados. 0s artigos que não apresentassem dados específicos sobre o uso de psicofármacos nos doentes com VIH foram excluídos. Foram ainda consultados cinco livros de texto que contemplam o tema e 0 site da OMS. Aborda-se em seguida o uso de antipsicóticos, antidepressivos, benzodiazepinas e estabilizadores do humor em doentes com VIH.

\section{Antipsicóticos em Doentes com VIH}

A prevalência de patologia psicótica de novo nos doentes com VIH/SIDA é pouco clara, as taxas variam entre 0,2 e $15 \%$.

0s distúrbios psicóticos classificam-se em primários, que ocorrem independentemente da infecção por VIH (cujo aparecimento pode ser prévio ao seu diagnóstico) e em secundários, que surgem como consequência da toxicidade do vírus ${ }^{20}$. No primeiro caso, o diagnóstico da infecção constitui um factor de stress, que em conjunto com uma certa vulnerabilidade genética e outros factores psicossociais, contribui para a descompensação psicótica. No segundo caso, são as alterações orgânicas no SNC, causadas pelo VIH, que podem desencadear um quadro clínico de psicose ${ }^{19}$, considerado secundário à infecção. 0 aparecimento de doença psicótica é mais prevalente nos estadios tardios da infecção, o que sustenta a hipótese da psicose ser um efeito directo da neurotoxicidade do VIH no $\mathrm{SNC}^{21}$. Por outro lado, os sintomas psicóticos podem surgir, ainda, associados à medicação anti-retrovírica, como efeito secundário de alguns fármacos. Por exemplo, na literatura foram descritos, em vários casos, síndromas maníacos associados à terapêutica com zidovudina ${ }^{22}$. Sintomas psicóticos, tais como alucinações acústico-verbais, confusão e alterações do humor, foram asso- 
ciados à terapêutica com ganciclovir ${ }^{22}$. Para além de ideação delirante, agitação e insónia, foram igualmente reportados sintomas confusionais em doentes medicados com efavirenz, sobretudo no período inicial de administração do fármaco ${ }^{22}$. Sintomas psicóticos foram ainda verificados numa doente medicada com combinação de abacavir, nevirapina, e combivir ${ }^{22}$. 0 tratamento sintomático consiste na toma de antipsicóticos (AP), devendo ser a primeira opção na fase aguda da doença ${ }^{23}$. Habitualmente 0 tratamento psicofarmacológico dos doentes com VIH é semelhante ao dos doentes sem infecção; contudo, deve ter-se em atenção os efeitos secundários dos mesmos, a necessidade de usar doses mais baixas que o habitual e a duração do tratamento ${ }^{13,24}$.

Nas patologias psicóticas e nas psicoses por estados comórbidos os AP atípicos são geralmente a escolha adequada. A neurotoxicidade do $\mathrm{VIH}$ torna os doentes mais sensíveis aos efeitos adversos dos AP convencionais, especialmente os efeitos extrapiramidais (EEP) também conhecidos por sintomas parkinsónicos ${ }^{25}$. Neste contexto, os AP atípicos causam menos EEP do que os AP tradicionais ${ }^{26-28}$, o que pode melhorar a adesão ao tratamento antipsicótico. No entanto, há que estar atento ao risco de dislipidémias e hiperglicémia nos doentes que já estão medicados com terapêutica anti-retroviral ${ }^{29-31}$.

De forma geral, para os AP surge resposta geralmente com $1 / 4$ a $1 / 2$ da dose necessária para indivíduos com esquizofrenia da mesma idade $^{18}$. Além disso, há múltiplas interações referidas na literatura ${ }^{32}$. Estas interações são moduladas maioritariamente por isoenzimas do citocromo P450 (CYP), particularmente a CYP2D6 e CYP3A.
Nalguns casos, a associação de anti-retrovirais com inibição da CYP - há, por exemplo, inibição de algumas isoformas da CYP pelo ritonavir - e determinados psicofármacos, promove um aumento da substância activa podendo causar toxicidade. É o caso da interação com risperidona, metabolizada pela CYP2D6 e também pela $3 \mathrm{~A}$, particularmente $3 \mathrm{~A} 4^{33}-35$, quetiapina, e alguns agentes ditos típicos, como a cloropromazina, o haloperidol e a pimozida $^{32}$.

Por outro lado, a associação de fármacos pode determinar uma diminuição da substância activa com repercussão na eficácia. 0 ritonavir diminui significativamente os níveis plasmáticos da olanzapina, por aumento da actividade da CYP1A2 e UDP-glucoronil-transferase $^{27}$. Este psicofármaco não tem interacção relevante com outros IP's ${ }^{34}$. Apesar do referido, deve ter-se atenção ao potencial para aumentos tóxicos com outros inibidores da CYP ${ }^{32}$. Foi igualmente descrito que a indução do CYP1A2 pelo ritonavir diminui a concentração de clozapina $^{33}$.

Por último, podem não se encontrar interacções conhecidas. É o caso da associação com amissulprida e ziprasidona, que têm poucos ensaios em doentes com VIH.

Os efeitos decorrentes das interações entre estes fármacos podem condicionar ajuste de dose ou mesmo contra-indicação. Nos casos em que a interação promove um aumento da substância activa a dose pode ter de ser reduzida ou a associação pode mesmo estar contra-indicada. A combinação de risperidona com NRTI's e IP's, particularmente com ritonavir e indinavir ${ }^{33}$, deve ser evitada. Na associação, 0 aumento dos níveis plasmáticos de risperi- 
dona pode potenciar a ocorrência de síndroma maligno dos neurolépticos ${ }^{32}$. No entanto, a administração de risperidona mostra-se eficaz e com menos efeitos secundários, nomeadamente EEP, que os neurolépticos convencionais e não é frequente a sua associação a agranulocitose ou convulsões ${ }^{15,34}$. Pode ser necessária redução da dose de haloperidol com ritonavir, dado que este fármaco tem uma incidência elevada de efeitos extra-piramidais potenciados pela interaçã $0^{8,33}$. A associação de inibidores da CYP com pimozida foi mesmo contra-indicada ${ }^{32}$. Além disso, o uso de pimozida deve igualmente ser vigiado ou mesmo evitado na associação com outros medicamentos que possam aumentar o intervalo QT e ter efeitos pró-arrítmicos ${ }^{8}$, por risco de toxicidade cardíaca ${ }^{33}$. Quando ocorre diminuição da substância activa na interação, a dose pode ter de ser aumentada ou a associação pode estar contra-indicada. Por exemplo, deve considerar-se 0 ajuste/aumento de dose na associação entre ritonavir e olanzapina ${ }^{33}$. A associação entre ritonavir e clozapina está contra-indicada $^{33,35}$. Note-se que a clozapina não está recomendada em doentes com VIH dado o risco de agranulocitose (1-2\%) ${ }^{34}$. Por último, há fármacos com ausência de interações conhecidas. A amissulprida, com eliminação renal quase exclusiva, é vantajosa em doentes com alterações hepáticas ${ }^{34}$. A ziprasidona é geralmente bem tolerada.

\section{Antidepressivos em Doentes com VIH}

A depressão major (DM) é a alteração psíquica mais frequente nos doentes infectados com $\mathrm{VIH}^{36}$, com uma prevalência significativamente maior do que a encontrada na população geral (cerca de 35\%) ${ }^{37}$. A depressão pode acelerar a progressão da infecção VIH para SIDA, pelo que o diagnóstico precoce e o tratamento adequado são a chave para evitar que isso aconteça ${ }^{38}$.

Foi mostrado em vários estudos que a medicação antidepressiva é eficaz no tratamento da depressão, na maioria dos doentes com $\mathrm{VIH}^{39}$. Entre os fármacos antidepressivos estudados em doentes com VIH, os antidepressivos tricíclicos (ADT) e os inibidores selectivos da recaptação da serotonina (ISRS) foram os mais relevantes. A eficácia destas duas classes de antidepressivos é considerada semelhante porém, com os ADT, o índice de abandono ao tratamento foi superior ${ }^{40}$. Em geral, os ADT são fármacos a evitar como primeira linha devido aos seus efeitos secundários anticolinérgicos e pelo seu poder sedativo ${ }^{41}$. Os ISRS podem ser os fármacos de eleição, pela menor incidência de efeitos adversos anticolinérgicos e antiadrenérgicos e pelo seu alto nível de segurança em caso de sobredosagem ${ }^{41}$. As terapêuticas anteriormente realizadas são também importantes pois se determinado fármaco já foi eficaz num doente é provável que o volte a ser ${ }^{42}$. Em seguida apresentam-se os principais ISRS, $\mathrm{ADT}$, Inibidores selectivos da recaptação da serotonina e da noradrenalina (ISRSN), e ainda o bupropiom, e descrevem-se, de modo sumário, as interacções com a terapêutica HAART e os efeitos adversos que daí possam advir.

ISRS - Inibidores Selectivos da Recaptação da Serotonina

Quanto aos ISRS destaca-se que os inibidores de protease (IP's) e a maioria dos ISRS são metabolizados por enzimas do sistema do cito- 
cromo P450 (CYP2D6). Visto estes anti-retrovirais inibirem a via enzimática que metaboliza o ISRS, vai haver um aumento dos níveis plasmáticos e dos efeitos secundários desse mesmo ISRS, podendo mesmo ocorrer síndroma serotoninérgico ${ }^{43,44}$. Para evitar tais complicações, deve iniciar-se 0 tratamento com doses baixas de ISRS $^{27}$.

0 citalopram é uma escolha frequente pois apresenta um efeito inibitório mínimo no sistema enzimático que metaboliza os anti-retrovirais, com poucas interações medicamentosas ${ }^{18,27}$, porém o Lopinavir/r (associação liponavir e ritonavir) e o ritonavir aumentam os seus níveis ${ }^{34}$. Já 0 escitalopram não tem aparentemente interação com ritonavirir ${ }^{27,32}$. A fluoxetina é considerado o mais eficaz dos ISRS no tratamento da depressão major em doentes infectados pelo $\mathrm{VIH}^{32,34}$, com relativamente poucos efeitos secundários e sem efeitos negativos no sistema imune em doentes com VIH deprimidos ${ }^{35,45}$. Porém, amprenavir, delavirdine, indinavir, lopinavir/r, nelfinavir, efavirenz, saquinavir, e, sobretudo, ritonavir aumentam os níveis de fluoxetina por inibição da CYP2D66 ${ }^{32}$, estando descritos casos de síndroma serotoninérgico decorrentes da interação entre esta e os três últimos ${ }^{27}$. A nevirapina reduz os níveis plasmáticos de fluoxetina ${ }^{34}$. Por outro lado, em combinação com ritonavir a fluoxetina pode aumentar a concentração daquele $^{32}$, não havendo à partida necessidade de ajuste de dos $\mathrm{e}^{35}$. A fluvoxamina é um inibidor potente do CYP1A2 ${ }^{32}$. Também o amprenavir, efavirenz, indinavir, lopinavir/ $\mathrm{r}$, nelfinavir, ritonavir e saquinavir aumentam os níveis de fluvoxamina ${ }^{34} ;$ a fluvoxamina diminui a eliminação da nevirapina e aumenta a con- centração dos IP's podendo causar toxicida$\mathrm{de}^{27,32}$. A paroxetina é eficaz no tratamento da depressão em doentes com VIH, e tem menos efeitos secundários que a imipramina ${ }^{15,27,32,35}$. Lopinavir/r e ritonavir também aumentam os níveis de paroxetina e esta aumenta a concentração dos IP's podendo causar toxicidade ${ }^{32,34}$. A sertralina, além de eficaz no tratamento da depressão em doentes com VIH ${ }^{27,35}$ é uma escolha frequente por efeito inibitório mínimo no sistema enzimático que metaboliza os anti-retrovirais, com poucas interações medicamentosas ${ }^{18}$. Está descrita uma interacção irrelevante com os IP's, particularmente lopinavir/r e ritonavir aumentam os seus níveis ${ }^{32}$.

Antidepressivos Tricíclicos (e Similares)

0 uso de ADT é mais tolerado na fase assintomática do que na fase avançada da infecção. Os doentes seropositivos tendem a ser mais sensíveis aos efeitos adversos dos ADT e, como tal, recomenda-se prudência na sua prescrição a estes doentes. Os ADT podem precipitar efeitos indesejáveis antihistaminérgicos e antiadrenérgicos ${ }^{35}$, para além dos expectáveis efeitos anticolinérgicos (incluindo alterações cognitivas, nalguns casos delirium, e ressecamento das mucosas, facilitando o desenvolvimento de candidíase $)^{46}$. No entanto, os ADT podem melhorar a diarreia e a insónia, sintomas comuns nestes doentes ${ }^{18}$. Caso se opte por um destes fármacos, é recomendado iniciar-se 0 tratamento com uma dose de cerca de 10-25 mg e ir aumentando gradualmente, consoante a tolerância do doente ${ }^{46}$.

A imipramina mostrou-se eficaz no tratamento da depressão em doentes com $\mathrm{VIH}^{15} \mathrm{e}$ sem efeitos negativos no sistema imune em 
doentes com VIH deprimidos ${ }^{45}$, embora conforme referido anteriormente, com mais efeitos secundários que a paroxetina ${ }^{15,27,32,35}$. Lopinavir/r, ritonavir aumentam os níveis de amitriptilina, clomipramina, e imipramina ${ }^{34}$. A mirtazapina tem interacções potenciais com os inibidores da CYP, como o ritonavir ${ }^{32}$. A trazodona aumenta potencialmente a dose com IP's, tal como o ritonavir, e NRTI's ${ }^{32}$. Na coadministração de trazodona e ritonavir aumenta a sedação, a fadiga, e a disfunção executiva $^{27}$, podendo ser necessária redução de dose $^{33}$. Em indivíduos saudáveis a adição de ritonavir resultou em náusea, tonturas, hipotensão, e num caso morte ${ }^{32}$.

ISRSN - Inibidores Selectivos da Recaptação da Serotonina e da Noradrenalina

Outros fármacos que têm efeito benéfico, quer na sintomatologia depressiva quer na dor, são os ISRSN. A dor é frequentemente subtratada nos doentes com VIH estando estabelecido que os ISRSN são agentes efectivos no tratamento da dor crónica ${ }^{27,32}$, embora não tenham sido encontrados estudos sobre os benefícios na neuropatia associada ao $\mathrm{VIH}^{47}$.

A venlafaxina é uma escolha frequente por efeito inibitório mínimo no sistema enzimático que metaboliza os anti-retrovirais, com poucas interações medicamentosas ${ }^{18,45}$; porém, diminui os níveis plasmáticos de indinavir ${ }^{27,32}$ e requer precaução se houver, entre outros, história de doença cardíaca ou de abuso de drogas $^{8}$. A duloxetina é eficaz e bem tolerada, porém pode aumentar a severidade de efeitos adversos quando combinado com inibidores potentes do CYP2D6 tal como o ritonavir ${ }^{32} \mathrm{e}$ pode causar elevação das enzimas hepáticas, requerendo precaução na prescrição a doentes com insuficiência hepática ou doentes coinfectados com VHC ${ }^{27}$.

\section{$\underline{\text { Outros }}$}

Vários outros fármacos têm sido usados no tratamento da depressão incluindo outros antidepressivos, estimulantes e terapêuticas hormonais ${ }^{27}$. Destaca-se o bupropiom por ser considerado eficaz e bem tolerado ${ }^{27,35,45}$. Porém, há aumento do risco de convulsões na interacção deste fármaco com ritonavir e efavirenz e pode ser necessário ajuste de dose $\mathrm{e}^{27,33}$; nomeadamente, aumento de dose de efavirenz e nevirapina e redução de dose de bupopriom ${ }^{33}$.

\section{Benzodiazepinas em Doentes com VIH}

A ansiedade e angústia são sentimentos frequentes em doentes com $\mathrm{VIH}^{48,49}$, tendo uma prevalência estimada de cerca de $38 \%{ }^{50}$. A ansiedade pode manifestar-se em diferentes momentos no decurso da infecção pelo VIH ${ }^{51}$.

0 modo como o doente manifesta a sua ansiedade depende de vários factores: da história pessoal, dos traços de personalidade e das suas próprias defesas ${ }^{52}$. A ansiedade acarreta um impacto negativo no tratamento dos doentes com VIH ${ }^{33,54}$. A divulgação da doença a outras pessoas, sobretudo se o doente pertencer a um grupo minoritário, está associada a maior ansiedade ${ }^{55,56}$. Caso o doente tenha um coping desadaptativo, a ansiedade poderá ser ainda maior, motivando a toma de ansiolíticos ${ }^{57}$.

As benzodiazepinas (BZ) têm, entre outras, uma acção ansiolítica e hipnótica. Em doentes com infecção VIH estas devem ser prescritas com precaução, sendo aconselhável seleccionar as de semi-vida curta cuja acção é mais 
rápida a reverter ${ }^{58}$. No entanto, dado que apresentam maior risco de dependência, o seu uso deve ser equacionado, sobretudo em doentes com história de comportamentos aditivos.

Apesar do uso de ansiolíticos ser associado a uma elevada adesão à terapêutica anti-retrovira $^{54}$, a sua toma continuada provoca dependência e dano cognitivo ${ }^{24,59}$. Além disso, juntar BZ aos anti-retrovirais pode resultar em interacções entre ambos ${ }^{24,60}$. As BZ são prioritariamente metabolizadas pela CYP3A4 ou por glucoronidação. Os IP's, bem como os NNRTI's, podem potenciar um risco aumentado de sedação severa e depressão respiratória na interacção com a maioria das BZ ${ }^{27,61}$. Como tal, a interacção entre BZ e IP's é um factor limitante da sua prescrição em doentes com VIH. Particularmente, o ritonavir não deve ser administrado com 0 alprazolam nem com midazolam, e triazolam ${ }^{33}$. Esse anti-retrovírico inibe a via enzimática do CYP3A e, por conseguinte, pequenas doses de ritonavir podem diminuir significativamente a metabolização das BZ referidas, aumentando o seu nível sérico e efeitos adversos incluindo morte ${ }^{27,62,63}$. Por outro lado, terapêuticas que induzem a glucoronidação, tal como ritonavir ou nelfinavir, podem reduzir os níveis de $\mathrm{BZ}$ metabolizadas por essa via, nomeadamente o lorazepam, oxazepam, e temazepam ${ }^{61}$.

\section{Estabilizadores de Humor em Doentes com VIH}

Episódios maníacos têm sido documentados associados ao $\mathrm{VIH}$, aumentando com a progressão da infecçã $0^{18}$. Nestes doentes, a mania pode surgir relacionada com uma doença bipolar, pode ser secundária aos efeitos neu- rotóxicos do VIH ou infecções secundárias ao VIH no SNC, ou pode ainda ser secundária à terapêutica anti-retroviral ${ }^{24,64}$. Entre os dois últimos, a neurotoxicidade é documentada como o factor mais importante da patogénese de mania secundária ao $\mathrm{VIH}^{64-67}$. Se não tratada a mania pode levar a um comportamento auto-destrutivo e à não adesão à terapêutica anti-retrovírica $^{68}$.

0 tratamento da doença bipolar inclui agentes estabilizadores de humor, como o carbonato de lítio, ácido valpróico, carbamazepina, lamotrigina, entre outros. 0s dois primeiros foram documentados por ter boa tolerância e melhorar a componente neuropsicológica ${ }^{69,70}$. 0 carbonato de lítio é o estabilizador do humor que tem menos potencial de ter interações específicas com anti-retrovirais ${ }^{32}$, embora possa não ser bem tolerado, sobretudo nos estadios avançados da infecção VIH, com sinais e sintomas de toxicidade ${ }^{15,18,45}$. Apesar de não haver alterações significativas na contagem de CD4, há diminuição acentuada da reacção linfocitária mista ${ }^{45}$. No entanto, não demonstrou influência no curso da infecção VIH melhorando a performance neuropsicológica ${ }^{27}$. A terapia com carbonato de lítio deve ser acompanhada de dosagem periódica dos níveis séricos de lítio, visando a manutenção de níveis terapêuticos $(0,5$ a $1,2 \mathrm{mEq} / \mathrm{l})$ e a prevenção de toxicidade (acima de $1,5 \mathrm{mEq} / \mathrm{l})^{71}$.

0 ácido valpróico pode diminuir o metabolismo da zidovudina mas sem relevância clínica conhecida ${ }^{32}$. Estudos infirmam interacção com efavirenz e lopinavir ${ }^{27}$. Hipoalbuminémia e coadministração de antibióticos durante 0 curso da doença VIH podem aumentar a concentração livre do ácido valpróico ${ }^{15}$. Estudos 
in vitro indicam que este pode promover a replicação viral; no entanto, os dados clínicos sugerem que tal não ocorre in vivo com terapêutica anti-retroviral adequada ${ }^{24,27}$. Requer monitorização dos níveis de ácido valpróico, enzimas hepáticas, CD4+, e carga viral ${ }^{27,32,45}$. Num estudo, houve aumento da replicação do citomegalovírus, mediador importante da replicação $\mathrm{VIH}^{35}$. 0 ácido valpróico foi ainda sugerido como tratamento adjuvante na disfunção cognitiva ${ }^{70}$.

Há indução da CYP3A pela carbamazepi$\mathbf{n a}^{33}$. A indução desta enzima aumenta o metabolismo de NTRI's ${ }^{15,45}$ e de IP's, tais como 0 indinavir ${ }^{27}$, podendo diminuir a sua dose $\mathrm{e}^{33,35}$. Num estudo houve diminuição dos níveis de indinavir com falência virológica pelos efeitos indutores da CYP da carbamazepina ${ }^{32}$. Por outro lado, ritonavir, e nelfinavir, aumentam 0 risco de toxicidade da carbamazepina por inibição do mesmo sistema enzimático ${ }^{15,27,32}$, ${ }^{35}$. A administração concomitante de ritonavir (IP) está contra-indicada, pois irá inibir a via enzimática CYP3A levando ao aumento do nível sérico de carbamazepina ${ }^{72}$; também não é recomendado o uso concomitante da carbamazepina com indinavir, saquinavir e nelfinavir ${ }^{35}$. Na associação com IP's e NNRTI's a monitorização terapêutica é recomendada ${ }^{33}$. Pode não ser bem tolerada nos doentes em estadio avançado, sobretudo com alterações neuroimagiológicas ou neuropsicológicas $^{18}$.

A lamotrigina mostrou-se eficaz no tratamento da dor neuropática ${ }^{27}$. Tanto o lopinavir e ritonavir diminuem os níveis plasmáticos de lamotrigina e tal requer ajuste de dose da lamotrigina $^{27}$, embora sem implicações clínicas ${ }^{32}$.

\section{DISCUSSÃO}

As alterações psiquiátricas em doentes com VIH são frequentes e têm sido conduzidos vários estudos que têm em consideração 0 respectivo uso de psicofármacos. Como já foi referido, estas surgem devido à presença de vias comuns de metabolização de fármacos, nomeadamente as das isoenzimas do sistema do citocromo $\mathrm{P} 450^{73}$.

0 ritonavir é 0 anti-retroviral que apresenta um maior número de interacções medicamentosas e com maior gravidade, dada a sua acção sobre várias enzimas de metabolização. 0 ritonavir e restantes IP's têm sido os mais estudados. Não se encontraram estudos sobre NRTI's à excepção da zidovudina nem sobre inibidores da fusão. Quanto aos psicofármacos, os antidepressivos e ansiolíticos, sedativos, e hipnóticos foram os mais estudados, seguindo-se os estabilizadores de humor. Há poucos estudos sobre as interacções dos anti-retrovirais com os antipsicóticos, apesar da referida (relativamente) elevada prevalência de psicose nesta população. Mais estudos nestas classes são necessários no sentido de haver mais indicações para uma boa prática clínica. Enquanto estes estudos não estão disponíveis assume-se a regra geral que no caso dos doentes medicados com anti-retrovirais, que necessitam de instituição de psicofármacos, deve começar-se com doses baixas e fazer aumentos paulatinos ${ }^{27}$.

Embora este estudo se tenha debruçado sobre os psicofármacos e VIH, importa salientar a relevância das intervenções psicoterapêuticas, quer isoladamente, quer combinadas com os regimes psicofarmacológicos, no tratamento dos doentes infectados pelo $\mathrm{VIH}^{35}$. 


\section{CONCLUSÃO}

0 tratamento da comorbilidade psiquiátrica é determinante na evolução dos doentes, quer no sentido de reduzir o sofrimento associado à vivência da infecção VIH/SIDA e das suas complicações, quer no sentido de melhorar a adesão à terapêutica e o prognóstico a longo prazo. Como tal, a escolha da intervenção terapêutica, nomeadamente dos psicofármacos, com o menor número de interacções e efeitos adversos é essencial para alcançar o sucesso terapêutico no combate da infecção.

\section{Conflitos de Interesse / Conflicting Interests:}

Os autores declaram não ter nenhum conflito de interesses relativamente ao presente artigo.

The authors have declared no competing interests exist.

\section{Fontes de Financiamento / Funding:}

Não existiram fontes externas de financiamento para a realização deste artigo.

The authors have declared no external funding was received for this study.

\section{Bibliografia / References}

1. Mandell GL, Bennett JE, Dolin R: Principles and practice of infectious diseases. 4th ed. New York: Ed. Churchill Livingstone; 1995.

2. Sande MA, Gilbert DN, Moellering RC: The Sanford: guia para o tratamento da Aids/HIV. 7 ed. Rio de Janeiro: EPUC Ed. de Publicações Médicas Ltda; 1998.

3. Veronesi R, Focaccia R: Tratado de Infectologia. São Paulo: Ed. Atheneu; 1997.

4. Stevens LM: HIV Infection: The Basics. JAMA. July 21, 2010, Vol 304, No. 3.
5. Organization WHO. Fact sheet $\mathrm{N}^{\circ} 360$. WHO; [updated June 2013 Retrieved 14 August 2013]; Available from: http://www.who.int/mediacentre/factsheets/fs360/en/.

6. Antiretroviral therapy for HIV infection in adults and adolescents: recommendations for a public health approach. 2010 revision ed. Geneva: World Health Organization; 2010.

7. Thompson MA, Aberg JA, Cahn P, Montaner JSG, Rizzardini G, Telenti A, et al: Antiretroviral treatment of adult HIV infection: 2010 recommendations of the International AIDS Society-USA panel. Jama. 2010 Jul;304(3):321-33.

8. Carmona M, Esteves AP, Gonçalves J, Macedo $\mathrm{T}$, Mendonça J, Osswald $\mathrm{W}$, et al.: Prontuário Terapêutico - 10. INFARMED - Autoridade Nacional do Medicamento e Profutos de Saúde, IP / Ministério de Saúde; Agosto de 2011.

9. Elzi L, Marzolini C, Furrer H, Ledergerber B, Cavassini M, Hirschel B, et al.: Treatment modification in human immunodeficiency virus-infected individuals starting combination antiretroviral therapy between 2005 and 2008 . Arch Intern Med. 2010 Jan 11;170(1):57-65.

10. Levi GC, Vitória MA: Fighting against AIDS: the Brazilian experience. Aids. 2002 Dec 6;16(18):2373-83.

11. Israelski DM, Prentiss DE, Lubega S, Balmas G, Garcia P, Muhammad M, et al.: Psychiatric co-morbidity in vulnerable populations receiving primary care for HIV/AIDS. AIDS Care. 2007 Feb;19(2):220-5.

12. Scaravilli F, Bazille C, Gray F: Neuropathologic contributions to understanding AIDS and the central nervous system. Brain Pathol. 2007 Apr;17(2):197-208. 
13. Treisman GJ, Kaplin AI: Neurologic and psychiatric complications of antiretroviral agents. Aids. 2002 Jun 14;16(9):1201-15.

14. Raines C, Radcliffe 0, Treisman GJ: Neurologic and psychiatric complications of antiretroviral agents. J Assoc Nurses AIDS Care. 2005 Sep-0ct;16(5):35-48.

15. Dubé B, Benton T, Cruess DG, Evans DL: Neuropsychiatric manifestations of HIV infection and AIDS. J Psychiatry Neurosci. 2005 Jul;30(4):237-46.

16. Turjanski N, Lloyd GG: Psychiatric side-effects of medications: recent developments. Advances in Psychiatric Treatment. 2005;11:58-70.

17. Sisca AAM, Porto AR, Santos JRB: Evaluation of drug interactions with antidepressants and antipsychotics in patient submitted to antiretroviral treatment. ConScientiae Saúde. 2009;8(2):345-351.

18. Hinkin CH, Castellon SA, Atkinson JH, Goodkin K. Neuropsychiatric aspects of HIV infection among older adults. J Clin Epidemiol. Dec 2001;54(Suppl 1):S44-52.

19. Sewell DD: Schizophrenia and HIV. Schizophr Bull. 1996;22(3):465-73.

20. Alciati A, Fusi A, Monforte AA, Coen M, Ferri A, Mellado A. New-onset delusions and hallucinations in patients infected with HIV. Journal of Psychiatry \& Neuroscience. 2001; 26(3):229-234.

21. de Ronchi D, Faranca I, Forti P, Ravaglia G, Borderi M, Manfredi R, et al.: Development of acute psychotic disorders and HIV-1 infection. Int J Psychiatry Med. 2000;30(2):173-83.

22. Foster R, Olajide D, Everall IP: Antiretroviral therapy-induced psychosis: case report and brief review of the literature. HIV Med. 2003 Apr;4(2):139-44.
23. Hoffmann C, Rockstroh JK, Kamps: HIV Medicine 2007. www.HIVMedicine.com. 15th Ed. Flying Publisher. 2007: 674.

24. Ferrando SJ: Psychopharmacologic treatment of patients with HIV/AIDS. Current Psychiatry Reports. 2009 Jun;11(3):235-42.

25. Singh D, Goodkin K: Psychopharmacologic treatment response of HIV-infected patients to antipsychotic medications: J Clin Psychiatry. 2007 Apr:68(4):631-2.

26. Dolder CR, Patterson TL, Jeste DV: HIV, psychosis and aging: past, present and future. Aids. 2004 Jan 1;18 Suppl 1:S35-42.

27. Repetto MJ, Petitto JM: Psychopharmacology in HIV-infected patients. Psychosom Med. 2008;70(5):585-92.

28. Farah A: Atypicality of Atypical Antipsychotics. Prim Care Companion J Clin Psychiatry. 2005; 7(6): 268-274.

29. Nasrallah HA, Newcomer JW: Atypical antipsychotics and metabolic dysregulation: evaluating the risk/benefit equation and improving the standard of care. J Clin Psychopharmacol. 2004 0ct;24(5 Suppl 1):S7-14.

30. Oh J, Hegele RA: HIV-associated dyslipidaemia: pathogenesis and treatment. Lancet Infect Dis. 2007 Dec;7(12):787-96.

31. Angelino AF, Treisman GJ. Issues in co-morbid severe mental illnesses in HIV infected individuals. Int Rev Psychiatry. 2008 Feb;20(1):95-101.

32. Thompson A, Silverman B, Dzeng L, Treisman G: Psychotropic medications and HIV. Clin Infect Dis. 2006 May 1;42(9):1305-10.

33. de Maat MM, Ekhart GC, Huitema AD, Koks $\mathrm{CH}$, Mulder JW, Beijnen JH. Drug interactions between antiretroviral drugs and co- 
medicated agents. Clin Pharmacokinet. 2003;42(3):223-82.

34. von Einsiedel RW, Berger T, Weisbrod M, Unverricht $S$, Hartmann M: [HIV patients with psychiatric illnesses. Treatment strategies and drug interactions]. Nervenarzt. 2001 Mar;72(3):204-15.

35. Ferrando SJ, Wapenyi K. Psychopharmacological treatment of patients with HIV and AIDS. Psychiatr Q. 2002 Spring;73(1):33-49.

36. Owe-Larsson B, Säll L, Salamon E, Allgulander C: HIV infection and psychiatric illness. Afr J Psychiatry (Johannesbg). 2009 May;12(2):115-28.

37. Treisman G, Angelino A: Interrelation between psychiatric disorders and the prevention and treatment of HIV infection. Clin Infect Dis. 2007;45 Suppl 4:S313-7.

38. Nelson JC: Anxious depression and response to treatment. Am J Psychiatry. 2008 Mar;165(3):297-9.

39. Himelhoch S, Medoff DR: Efficacy of antidepressant medication among HIV-positive individuals with depression: a systematic review and meta-analysis. AIDS Patient Care STDS. 2005 Dec;19(12):813-22.

40. Caballero J, Nahata MC: Use of selective serotonin-reuptake inhibitors in the treatment of depression in adults with HIV. Ann Pharmacother. 2005 Jan;39(1):141-5. Epub 2004 Nov 23.

41. van der Lee MJ, Blenke AA, Rongen GA, Verwey-van Wissen CP, Koopmans PP, Pharo C, et al.: Interaction study of the combined use of paroxetine and fosamprenavir-ritonavir in healthy subjects. Antimicrob Agents Chemother. 2007 Nov;51(11):4098-104.

42. Ebert. D: Psychiatrie systematisch. 4ed. Bremen: UNI-MED Verlag AG; 2001.
43. DeSilva KE, Le Flore DB, Marston BJ, Rimland D. Serotonin syndrome in HIV-infected individuals receiving antiretroviral therapy and fluoxetine. AIDS. $2001 \mathrm{Jul}$ 6;15(10):1281-5.

44. Tseng AL, Foisy MM: Significant interactions with new antiretrovirals and psychotropic drugs. Ann Pharmacother. 1999 Apr;33(4):461-73.

45. Chandra PS, Desai G, Ranjan S: HIV \& psychiatric disorders. Indian J Med Res. 2005 Apr;121(4):451-67.

46. Pieper AA, Treisman GJ: Drug treatment of depression in HIV-positive patients: safety considerations. Drug Saf. 2005;28(9):753-62.

47. Finnerup NB, Otto M, McQuay HJ, Jensen TS, Sindrup SH: Algorithm for neuropathic pain treatment: an evidence based proposal. Pain. 2005 Dec 5;118(3):289-305.

48. Bing EG, Burnam MA, Longshore D, Fleishman JA, Sherbourne CD, London AS, et al.: Psychiatric disorders and drug use among human immunodeficiency virus-infected adults in the United States. Arch Gen Psychiatry. 2001 Aug;58(8):721-8.

49. Basu S, Chwastiak LA, Bruce RD: Clinical management of depression and anxiety in HIV-infected adults. Aids. 2005 Dec 2;19(18):2057-67.

50. Whetten K, Reif S, Whetten R, Murphy-McMillan LK: Trauma, mental health, distrust, and stigma among HIV-positive persons: implications for effective care. Psychosom Med. 2008 Jun;70(5):531-8.

51. Gonzalez A, Solomon SE, Zvolensky MJ, Miller CT: The interaction of mindful-based attention and awareness and disengagement coping with HIV/AIDS-related stigma in regard to concurrent anxiety and depressive symptoms among adults with HIV/AIDS. J Health Psychol. 2009 Apr;14(3):403-13. 
52. Bravo P, Edwards A, Rollnick S, Elwyn G: Tough decisions faced by people living with HIV: a literature review of psychosocial problems. AIDS Rev. 2010 Apr-Jun;12(2):76-88.

53. Campos LN, Guimaraes MD, Remien RH: Anxiety and depression symptoms as risk factors for non-adherence to antiretroviral therapy in Brazil. AIDS Behav. 2010 Apr;14(2):289-99.

54. Roux P, Carrieri MP, Michel L, Fugon L, Marcellin F, Obadia Y, et al.: Effect of anxiety symptoms on adherence to highly active antiretroviral therapy in HIV-infected women. J Clin Psychiatry. 2009 Sep;70(9):1328-9.

55. Hatzenbuehler ML: How does sexual minority stigma "get under the skin"? A psychological mediation framework. Psychol Bull. 2009 Sep;135(5):707-30.

56. Roux P, Fugon L, Michel L, Lert F, Obadia Y, Spire B, et al.: Determinants of benzodiazepine use in a representative population of HIV-infected individuals: the role of HIV status disclosure (ANRS-EN12-VESPA study). AIDS Care. 2011 Sep;23(9):1163-70.

57. Serovich JM, Kimberly JA, Mosack KE, Lewis TL: The role of family and friend social support in reducing emotional distress among HIV-positive women. AIDS Care. 2001 Jun;13(3):335-41.

58. Golub SA, Tomassilli JC, Parsons JT: Partner serostatus and disclosure stigma: implications for physical and mental health outcomes among HIV-positive adults. AIDS Behav. 2009 Dec;13(6):1233-40.

59. Barker MJ, Greenwood KM, Jackson M, Crowe SF: Persistence of cognitive effects after withdrawal from long-term benzodiazepine use: a meta-analysis. Arch Clin Neuropsychol. 2004 Apr;19(3):437-54.
60. Malbergier A, Schöffelc AC: Tratamento de depressão em indivíduos infectados pelo HIV. Revista Bras Psiquiatr. 2001;3(23):160-7.

61. Antoniou T, Tseng AL: Interactions between recreational drugs and antiretroviral agents. Ann Pharmacother. 2002 0ct;36(10):1598-613.

62. Chouinard G. Issues in the clinical use of benzodiazepines: potency, withdrawal, and rebound. J Clin Psychiatry. 2004;65 Suppl 5:7-12.

63. Wynn GH, Cozza KL, Zapor MJ, Wortmann GW, Armstrong SC: Med-psych drug-drug interactions update. Antiretrovirals, part III: antiretrovirals and drugs of abuse. Psychosomatics. 2005 Jan-Feb;46(1):79-87.

64. Nakimuli-Mpungu E, Musisi S, Mpungu SK, Katabira E: Primary mania versus HIV-related secondary mania in Uganda. Am J Psychiatry. 2006 Aug;163(8):1349-54; quiz 480.

65. Ellen SR, Judd FK, Mijch AM, Cockram A: Secondary mania in patients with HIV infection. Aust N Z J Psychiatry. 1999 Jun;33(3):353-60.

66. Mijch AM, Judd FK, Lyketsos CG, Ellen S, Cockram A: Secondary mania in patients with HIV infection: are antiretrovirals protective? The Journal of neuropsychiatry and clinical neurosciences. 1999 Fall;11(4):475-80.

67. Lyketsos CG, Hanson AL, Fishman M, Rosenblatt A, McHugh PR, Treisman GJ: Manic syndrome early and late in the course of HIV. Am J Psychiatry. 1993 Feb;150(2):326-7.

68. Beyer JL, Taylor L, Gersing KR, Krishnan KR: Prevalence of HIV infection in a general psychiatric outpatient population. Psychosomatics. 2007 Jan-Feb;48(1):31-7.

69. Letendre SL, Woods SP, Ellis RJ, Atkinson JH, Masliah E, van den Brande G, et al.: Lithium improves HIV-associated neurocognitive impairment. Aids. 2006 Sep 11;20(14):1885-8. 
70. Schifitto G, Peterson DR, Zhong J, Ni H, Cruttenden K, Gaugh M, et al.: Valproic acid adjunctive therapy for HIV-associated cognitive impairment: a first report. Neurology. 2006 Mar 28;66(6):919-21.

71. Gallant J, Pham PA: Johns Hopkins HIV Guide 2012. Burlington. Jones \& Bartlett Learning. 2012: 122-123.

72. Berbel Garcia A, Latorre Ibarra A, Porta Etessam J, Martinez Salio A, Perez Martinez D,
Siaz Diaz R, et al: Protease inhibitor-induced carbamazepine toxicity. Clin Neuropharmacol. 2000 Jul-Aug;23(4):216-8.

73. American Psyciatric Association: Practice guidelines for the treatment of patients with HIV/ AIDS. Washington DC. American Psyciatric Association. 2000. 